\title{
Fiberoptic Cleaver Device
}

National Cancer Institute

\section{Source}

National Cancer Institute. Fiberoptic Cleaver Device. NCI Thesaurus. Code C50349.

A device designed for cutting an optical fiber and processing the ends for attachment. 